\title{
Absceso cerebral por Cladophialophora bantiana en un paciente con trasplante renal: reporte de un caso
}

John Fredy Nieto-Ríos ${ }^{1,2}$, Douglas Ramón Villafañe-Bermúdez ${ }^{3}$, Gustavo Adolfo Guerrero-Tinoco ${ }^{3}$, Isabel Cristina Ramírez-Sánchez ${ }^{2,4}$, Lina María Serna-Higuita ${ }^{5}$, Arbey Aristizábal-Alzate $^{1}$, Catalina Ocampo-Kohn ${ }^{1,2}$, Gabriel Varela ${ }^{6}$, Gustavo Zuluaga-Valencia ${ }^{1}$

${ }^{1}$ Departamento de Nefrología y Trasplante Renal, Hospital Pablo Tobón Uribe, Medellín, Colombia

${ }^{2}$ Departamento de Medicina Interna, Universidad de Antioquia, Medellín, Colombia

${ }^{3}$ Departamento de Nefrología Pediátrica, Universidad de Antioquia, Medellín, Colombia

${ }^{4}$ Departamento de Infectología, Hospital Pablo Tobón Uribe, Medellín, Colombia

${ }^{5}$ Institute for Clinical Epidemiology and Applied Biometrics, Eberhard Karls University, Tübingen, Germany

${ }^{6}$ Departamento de Patología, Hospital Pablo Tobón Uribe, Medellín, Colombia

Las feohifomicosis cerebrales son infecciones graves causadas por mohos dematiáceos, entre los cuales Cladophialophora bantiana es una de las especies más comúnmente aislada. Esta tiene tropismo por el sistema nervioso central y frecuentemente produce abscesos cerebrales en pacientes inmunocompetentes; además, en los inmunocomprometidos también puede ocasionar infección diseminada.

Pese a la disponibilidad de medicamentos antifúngicos de amplio espectro, a menudo se requiere también la intervención quirúrgica; de todas maneras, la mortalidad es elevada. El diagnóstico debe hacerse interviniendo para tomar la muestra y hacer el cultivo y las pruebas de sensibilidad.

Se presenta aquí el caso de un paciente con trasplante renal que presentó un absceso cerebral por $C$. bantiana, el cual se extrajo mediante resección quirúrgica. El paciente recibió tratamiento con voriconazol, con adecuada respuesta, mejoría y sin secuelas neurológicas.

Palabras clave: feohifomicosis; hongos; inmunosupresión; trasplante de riñón; absceso cerebral.

\section{Brain abscess caused by Cladophialophora bantiana after renal allograft loss: A case report}

Cerebral feohifomycosis are severe infections caused by dematiaceous fungi. Cladophialophora bantiana is one of the most commonly isolated species; it has central nervous system tropism and it often manifests as a brain abscess in immunocompetent patients. In immunocompromised patients, it can lead to brain abscesses and disseminated infections. Despite the availability of broad-spectrum antifungal drugs, it is a must to perform surgical management, in addition to drug therapy. However, mortality is high. The diagnostic approach must be invasive to establish a timely diagnosis and direct treatment based on culture and susceptibility tests.

Nieto-Ríos JF, Villafañe-Bermúdez DR, GuerreroTinoco GA, Ramírez-Sánchez IC, Serna-Higuita LM, Aristizábal-Alzate A, et al. Absceso cerebral por Cladophialophora bantiana en un paciente con trasplante renal: reporte de un caso. Biomédica. 2019;39(Supl.2):20-25

https://doi.org/10.7705/biomedica.v39i3.4471

\section{Correspondencia:}

John Fredy Nieto-Ríos, Departamento de Nefrología y Trasplante Renal, Hospital Pablo Tobón Uribe,

Calle 78 B N 69-240, Medellín, Colombia

Teléfono: (574) 4459902

johnfredynieto@gmail.com; Im.serna@hotmail.com

Contribución de los autores:

Todos los autores contribuyeron en la concepción de estudio, el diseño del manuscrito y la adquisición y e análisis e interpretación de los datos, así como en la redacción del manuscrito.

Financiación:

Este trabajo fue financiado por el Hospital Pablo Tobón Uribe.

Conflicto de intereses:

Los autores declaran que no tienen conflictos de intereses relacionados con los contenidos de este artículo.
We report a case of brain abscess caused by $C$. bantiana in an immunosuppressed patient who was treated with surgical resection and voriconazole with an adequate response to therapy and without neurological sequels.

Keywords: Phaeohyphomycosis; fungi; immunosuppression; kidney transplantation; brain abscess.

'Feohifomicosis' es el término empleado para denominar las infecciones causadas por mohos que contienen melanina en su pared celular, los cuales son ubicuos en la naturaleza y afectan tanto a los humanos como a los animales. Aunque muchas especies se consideran oportunistas, algunos son verdaderos agentes patógenos cuyo principal factor de virulencia es la producción de melanina, lo que les permite evadir el sistema inmunológico (1).

El espectro de la enfermedad es amplio, pues ocasiona desde infecciones localizadas hasta la enfermedad invasiva, independientemente del estado inmunológico de la persona. Son la primera causa de abscesos cerebrales de etiología fúngica en pacientes inmunocompetentes, y se consideran emergentes en los pacientes inmunosuprimidos. Son hongos filamentosos pigmentados, que contienen elementos miceliales tabicados y melanina de dihidroxinaftaleno en su pared celular, la cual le confiere el color oscuro a sus conidias e hifas (1-4). 
Se presenta el caso de un paciente con trasplante renal que desarrolló un absceso cerebral por Cladophialophora bantiana y requirió tratamiento quirúrgico y antifúngico, con lo cual mejoró adecuadamente.

\section{Caso clínico}

Se trata de un ingeniero agropecuario de 54 años de edad, con antecedentes de enfermedad renal crónica terminal asociada con nefrolitiaisis bilateral de causa indeterminada en esa época, por lo cual se encontraba en tratamiento con diálisis peritoneal desde mayo de 2008.

Fue remitido al Hospital Pablo Tobón Uribe para trasplante renal, el cual se realizó de donante fallecido en julio de 2010. Recibió tratamiento de inducción con alemtuzumab y metilprednisolona, y tratamiento de mantenimiento con ciclosporina, micofenolato de mofetilo y prednisolona. En noviembre de 2011, el paciente presentó disfunción del injerto renal, por lo que se le hizo una biopsia que evidenció un rechazo celular de categoría 2A según la clasificación de Banff; se trató con timoglobulina y esteroides, y se cambió la ciclosporina por tacrolimus.

Incidentalmente, en la biopsia también se reportaron infiltrados extensos por oxalato, cuyo estudio reveló concentraciones elevadas de oxalato en sangre y orina. Por tal motivo, se le practicó un estudio genético y se documentó la presencia de una hiperoxaluria primaria de tipo 2 que no se había diagnosticado previamente (5), lo cual significaba que sus riñones nativos se habían dañado por esta condición y que lo que presentaba era una recaída de dicha enfermedad en el riñón trasplantado.

Entonces, se le prescribió una dieta baja en oxalato, piridoxina, citrato de potasio e hidroclorotiacida; a pesar del tratamiento integral, presentó deterioro progresivo de la función renal hasta perder el injerto en mayo de 2015, por lo cual se desmontó la inmunosupresión paulatinamente y se inició la hemodiálisis.

A partir de esa fecha, incluso cuando todavía estaba bajo tratamiento inmunosupresor, el paciente comenzó a presentar fiebre intermitente, cefalea persistente, insomnio, vértigo y agitación psicomotora. Se le practicó una tomografía cerebral, la cual evidenció una lesión de 36 × 43 mm que ocupaba espacio en el hemisferio cerebeloso izquierdo y sugería la presencia de un absceso cerebral (figura 1).

El paciente fue sometido a craneotomía para drenar el contenido oscuro, denso, y hacer una resección completa de las lesiones; a continuación, se inició el tratamiento antimicrobiano con meropenem, vancomicina, metronidazol y anfotericina $B$ liposómica.

En el cultivo con agar Sabouraud, se obtuvo crecimiento de un moho negro identificado como C. bantiana (figura 2).

La resonancia magnética de control demostró que la resección había sido completa, con una zona de encefalomalacia residual. Con base en el diagnóstico histológico, se decidió suspender el tratamiento antibiótico y continuar con la administración de anfotericina $\mathrm{B}$ liposómica durante dos semanas y, posteriormente, con $200 \mathrm{mg}$ de voriconazol oral cada 12 horas durante 12 meses, obteniéndose una evolución adecuada.

Se inició la rehabilitación neurológica temprana y, a los tres años de seguimiento, el paciente se encontraba libre de la micosis, seguía con la terapia de reemplazo renal y no tenía secuelas neurológicas. Actualmente, 
el paciente se ha sometido al protocolo para trasplante de hígado y riñón, el cual es el tratamiento de elección para la hiperoxaluria primaria con enfermedad renal crónica terminal.

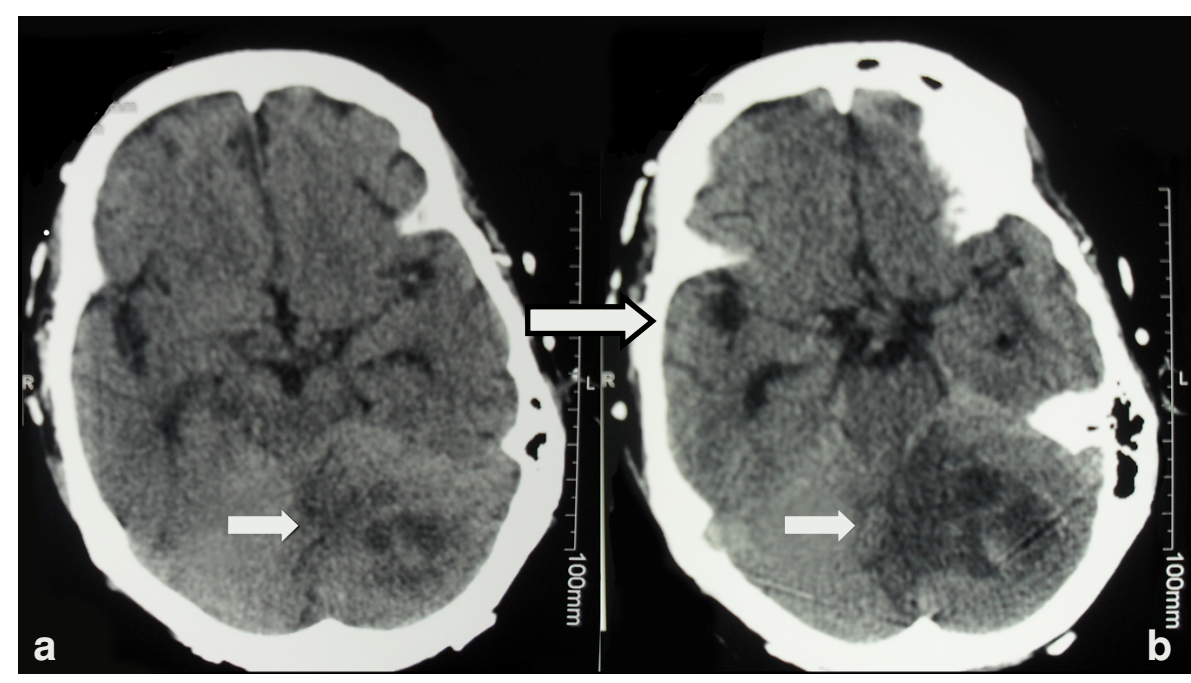

Figura 1. Dos cortes secuenciales (a y b) de la tomografía computarizada de cráneo. Se observa lesión de $36 \times 43 \mathrm{~mm}$ en el hemisferio cerebeloso izquierdo, sugestiva de absceso cerebral (flecha).

Imágenes originales reproducidas con autorización del Comité de Ética del Hospital Pablo Tobón Uribe

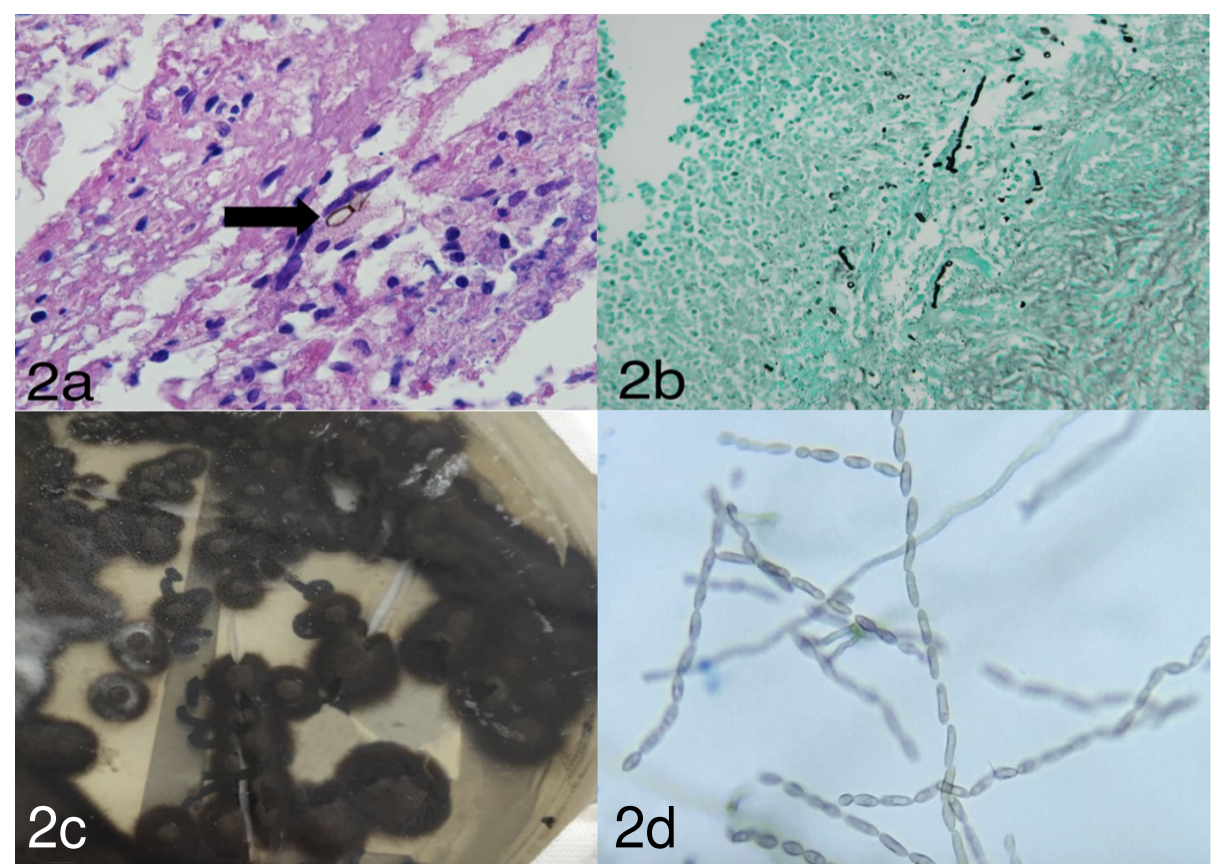

Figura 2. a. La flecha muestra una hifa tabicada. Hematoxilina y eosina, 40X. b. Se observan estructuras fúngicas filamentosas. Plata metenamina, 20X. c. Cultivo en agar Sabouraud a $30^{\circ} \mathrm{C}$ a las tres semanas de incubación: se observa crecimiento de moho negro. d. Presencia de conidias ovales que forman cadenas, características de C. bantiana. Microscopía de luz. 40X Imágenes originales reproducidas con autorización del Comité de Ética del Hospital Pablo Tobón Uribe 


\section{Discusión}

La feohifomicosis cerebral es el término empleado para las infecciones del sistema nervioso central ocasionadas por mohos dematiáceos (1). La mayoría de estos pertenecen a un orden único de hongos medioambientales, el de los Chaetothyriales; otros pocos están distribuidos en los órdenes Pleosporales, Sordariales, Xylariales, Helotiales y Botryosphaeriales (2). C. ladophialophora bantiana es una de las especies más comúnmente aislada en seres humanos y es responsable de la mayoría de los reportes de feohifomicosis cerebral (6) dado su neurotropismo (7), con una alta mortalidad debido al diagnóstico tardío y a la dificultad del tratamiento antifúngico $(7,8)$. Este agente patógeno es de distribución mundial, aunque las infecciones son más comunes en regiones subtropicales y en zonas climáticas no áridas $(1,3)$.

Aproximadamente, la mitad de los pacientes con feohifomicosis cerebral son inmunocompetentes (1-4). Sin embargo, es una condición emergente en receptores de trasplantes de órganos sólidos en pacientes con enfermedad renal crónica, neoplasias malignas, inmunodeficiencias primarias y secundarias, y con uso crónico de esteroides $(1,3,4,7,9,10)$.

Este fue el caso del paciente de este reporte, quien se encontraba aún bajo el efecto acumulado de los inmunosupresores, a lo cual se sumó la uremia por pérdida del injerto renal. Estos agentes patógenos alcanzan el sistema nervioso central a través de los vasos sanguíneos y linfáticos, por contigüidad a lesiones adyacentes o por inoculación accidental $(1,7,11,12)$. En este caso, se consideró que había adquirido el hongo por inhalación, ya que el paciente no había tenido trauma previo y con frecuencia visitaba zonas rurales no áridas por su trabajo.

La feohifomicosis puede producir afección local y comprometer la piel o los tejidos blandos, o puede ser invasiva con compromiso sistémico, incluido el sistema nervioso central $(9,13-16)$. El absceso cerebral es la complicación más grave y de mayor morbimortalidad $(4,6)$. Su presentación clínica incluye cefalea de aparición insidiosa, cambios en el comportamiento, convulsiones, fiebre de bajo grado y deficiencia neurológica focal $(3,4,8,12,17,18)$.

El diagnóstico temprano de la feohifomicosis cerebral permite la intervención terapéutica oportuna y la prevención de secuelas neurológicas graves. Para esto se requiere una alta sospecha clínica, obtener una imagen cerebral y estudiar las lesiones con biopsia estereotáxica o mediante la resección quirúrgica. La tomografía computarizada y la resonancia magnética son importantes ayudas en la detección de la infección y en el seguimiento terapéutico $(12,19)$.

Hay cuatro piedras angulares en el manejo de las infecciones por mohos del sistema nervioso central: el diagnóstico temprano, la administración de antifúngicos, la intervención neuroquirúrgica y el manejo de las secuelas neurológicas con rehabilitación (12). La resección completa de las lesiones es crucial en el manejo de la infección, ya que disminuye el inóculo y facilita la penetración del antifúngico al tejido comprometido, lo cual mejora el pronóstico $(1,2,4,12,19)$. El paciente de este caso fue intervenido quirúrgicamente de manera temprana, lo que favoreció el control de la infección y limitó las secuelas neurológicas. 
El tratamiento antifúngico debe orientarse con base en las pruebas de sensibilidad, el control de los niveles séricos alcanzados y la reacción clínica. Los antifúngicos con actividad contra este tipo de mohos son los polienos (formulaciones de anfotericina B), los triazoles (voriconazol, itraconazol y posaconazol), y la 5 -fluocitosina $(1,4,11,20)$. Cuando la reacción terapéutica no es la adecuada, se recomienda la combinación de antifúngicos.

En el caso reportado, el tratamiento antifúngico inicial consistió en anfotericina $\mathrm{B}$ liposómica durante dos semanas, con posterior cambio a voriconazol de $200 \mathrm{mg}$ cada 12 horas durante un año, con lo cual hubo una adecuada respuesta clínica y en los exámenes paraclínicos, con mínimas secuelas después de tres años de seguimiento. En este caso, no se hizo seguimiento de los niveles séricos de los antifúngicos.

En conclusión, la presencia de feohifomicosis cerebral debe sospecharse en todo paciente con síntomas neurológicos y lesión que ocupe espacio en el sistema nervioso central, de apariencia macroscópica oscura, y crecimiento de moho negro en los cultivos para hongos. Debe intentarse identificar la especie y hacer las pruebas de sensibilidad para orientar de manera adecuada el tratamiento antifúngico, el cual debe ser temprano y, en la medida de lo posible, incluir el manejo quirúrgico para lograr el control de la infección y minimizar las secuelas neurológicas.

\section{Agradecimientos}

Al Hospital Pablo Tobón Uribe por permitirnos realizar el reporte del caso.

\section{Referencias}

1. Li DM, de Hoog GS. Cerebral phaeohyphomycosis--a cure at what lengths? Lancet Infect Dis. 2009;9:376-83. https://doi.org/10.1016/\$1473-3099(09)70131-8

2. de Hoog G, Guarro J, Gene J, Figueras M. Atlas of clinical fungi. Second edition. Utrecht: Centraalbureau voor Schimmelcultures; 2001. p. 51-2.

3. Kantarcioglu AS, de Hoog GS. Infections of the central nervous system by melanized fungi: A review of cases presented between 1999 and 2004. Mycoses. 2004;47:4-13. https://doi.org/10.1046/j.1439-0507.2003.00956.x

4. Revankar SG, Sutton DA, Rinaldi MG. Primary central nervous system phaeohyphomycosis: A review of 101 cases. Clin Infect Dis. 2004;38:206-16. https://doi.org/10.1086/380635

5. Nieto-Ríos JF, Zuluaga M, Higuita LM, Flórez A, Bello-Márquez DC, Aristizábal A, et al. Primary hiperoxaluria diagnosed after kidney transplantation: Report of 2 cases and literature review. J Bras Nefrol. 2017;39:462-6. https://doi.org/ 10.5935/0101-2800.20170081

6. Hospenthal DR. Uncommon fungi and related species. In: Bennett JE, Dolin R, Blaser MJ, editors. Mandell, Douglas, and Bennett's Principles and Practice of Infectious Disease. Eight edition. Philadelphia, PA: Saunders; 2015. p. 3003-15. https://doi.org/10.1016/C2012-1-00075-6

7. Chakrabarti A, Kaur H, Rudramurthy SM, Appannanavar SB, Patel A, Mukherjee KK, et al. Brain abscess due to Cladophialophora bantiana: A review of 124 cases. Med Mycol. 2016;54:111-9. https://doi.org/ 10.1093/mmy/myv091

8. Huang WM, Fan YM, Li W, Yang WW. Brain abscess caused by Cladophialophora bantiana in China. J Med Microbiol. 2011;60:1872-4. https://doi.org/ 10.1099/jmm.0.032532-0

9. Nampoory MR, Khan ZU, Johny KV, Constandi JN, Gupta RK, Al-Muzairi I, et al. Invasive fungal infections in renal transplant recipients. J Infect. 1996;33:95-101. https://doi.org/10.1016/S0163-4453(96)92986-2

10. Shoham S. Emerging fungal infections in solid organ transplant recipients. Infect Dis Clin North Am. 2013;27:305-16. https://doi.org/10.1016/j.idc.2013.02.004

11. Centers for Disease Control and Prevention. Exophiala infection from contaminated injectable steroids prepared by a compounding pharmacy--United States, July-November 2002. MMWR Morb Mortal Wkly Rep. 2002;51:1109-12. 
12. McCarthy M, Rosengart A, Schuetz AN, Kontoyiannis DP, Walsh TJ. Mold infections of the central nervous system. N Engl J Med. 2014;371:150-60. https://doi.org/ 10.1056/NEJMra1216008

13. Queiroz-Telles F, Esterre P, Pérez-Blanco M, Vitale RG, Salgado CG, Bonifaz A. Chromoblastomycosis: An overview of clinical manifestations, diagnosis and treatment. Med Mycol. 2009;47:3-15. https://doi.org/10.1080/13693780802538001

14. Schoeffler A, Redon E, Contet-Audonneau N, Cuny JF, Lo-Jeanpierre B, Beurey P, et al. Cutaneous phaeohyphomycosis due to Cladophialophora bantiana. Ann Dermatol Venereol. 2011;138:504-7. https://doi.org/10.1016/j.annder.2011.01.044

15. Silveira ER, Resende MA, Mariano VS, Coura WA, Alkmim LD, Vianna LB, et al. Brain abscess caused by Cladophialophora (Xylohypha) bantiana in a renal transplant patient. Transpl Infect Dis. 2003;5:104-7. https://doi.org/10.1034/j.1399-3062.2003.00020.x

16. Silveira F, Nucci M. Emergence of black moulds in fungal disease: Epidemiology and therapy. Curr Opin Infect Dis. 2001;14:679-84.

17. Harrison DK, Moser S, Palmer CA. Central nervous system infections in transplant recipients by Cladophialophora bantiana. South Med J. 2008;101:292-6. https://doi.org/ 10.1097/SMJ.0b013e318164e289

18. Ben-Ami R, Lewis RE, Raad, II, Kontoyiannis DP. Phaeohyphomycosis in a tertiary care cancer center. Clin Infect Dis. 2009;48:1033-41. https://doi.org/10.1086/597400

19. Jacobs CS, Etherton MR, Lyons JL. Fungal infections of the central nervous system. Curr Infect Dis Rep. 2014;16:449. https://doi.org/10.1007/s11908-014-0449-2

20. Roche M, Redmond RM, O'Neill S, Smyth E. A case of multiple cerebral abscesses due to infection with Cladophialophora bantiana. J Infect. 2005;51:e285-8. https://doi.org/10.1016/j.jinf.2005.02.029 